\title{
Study on the Inheritance of Modern Architecture to Urban History Humanities by Taking Museums as Cases
}

\author{
Ding Feng \\ Shanghai Institute of Visual Arts
}

Keywords: city style, museum architecture, inheritance of history humanities, design intention

\begin{abstract}
The long-standing historical and cultural heritage and the humanistic style of "integration pluralism" are the great wealth of the Chinese nation. The rapid development of urbanization in China in recent years has seriously damaged the traditional scene of many cities. As a city card, museum is a window to show people the history and culture of the city. Combined with the problems of urban characteristic style and historical humanistic loss in the process of modern urbanization, this paper will take the museum building as a case to analyze and explore the design idea and intention communication of modern architecture inheriting urban history humanities.
\end{abstract}

Our country has a long history, vast territory and many nationalities, so the traditional culture presents the style of "integration pluralism", which is also a major feature of Chinese history humanities. In modern architecture, the structures, materials and technologies that have an impact on the shape and state of the building have all been globalized, and the main impact of which is that it weaken the folklore characteristics and local attributes of the culture to a large extent. When reflected in the field of cities and architecture, it leads to the architectural similarities in different cities and the demise of the unique features of the city ${ }^{[1]}$.

With the rapid development of urbanization in recent years, it is really a pity to see that the historical features of many cities in China have been destroyed, and more towns have become "the same face". In the process of moving towards a modern city, the city itself has gradually lost its extremely important attribute, that is, the city's history humanities, which has experienced the precipitation of years. However, architecture has the ideology to express the national connotation and the special ability to use space to depict culture, which determines that architecture can be used as the carrier of the inheritance of urban history humanities.

Museums have always been regarded as the city card and the key component of the city humanities. In a sense, the museum is even regarded as the cultural symbol of a city. The social and cultural attributes of the museum architecture make it often defined as the civilization symbol and humanistic image of a region or city, representing the spirit of the nation and the excellent cultural tradition. Because of the unique humanistic connotation of the museum, it has become an important urban human landscape, which also determines the important position of its urban cultural center ${ }^{[2]}$. Combined with the problems of urban features and historical and cultural loss in the process of modern urbanization, this paper will take museum architecture as a case to analyze and explore the design concept and intention communication of modern architecture to inherit urban history 
humanities.

\section{Jixi Museum ${ }^{[3]}$ : expressing traditional intentions in modern architecture languages}

Jixi Museum is located in the north of the ancient city of Jixi County, Anhui Province, the base site of which was used as the county Yamun and county government courtyard successively and was converted into a museum in the protection and repair of the overall planning of the ancient city. The overall planning of the museum echoes the urban texture of Jixi ancient city. Several building bodies with different volumes are covered by entire excessive roofing, and the tortuous and coherent roof shape looks like the natural landscape form around the base. The morphological layout of the building is designed to make the indoor and outdoor space comfortable and pleasant, so that it retains and uses the status of ancient and famous trees in the land as far as possible, and remolding the traditional space layout of Hui style architecture through the settings of a number of courtyard, patios and streets ${ }^{[4]}$. (Figure 1)

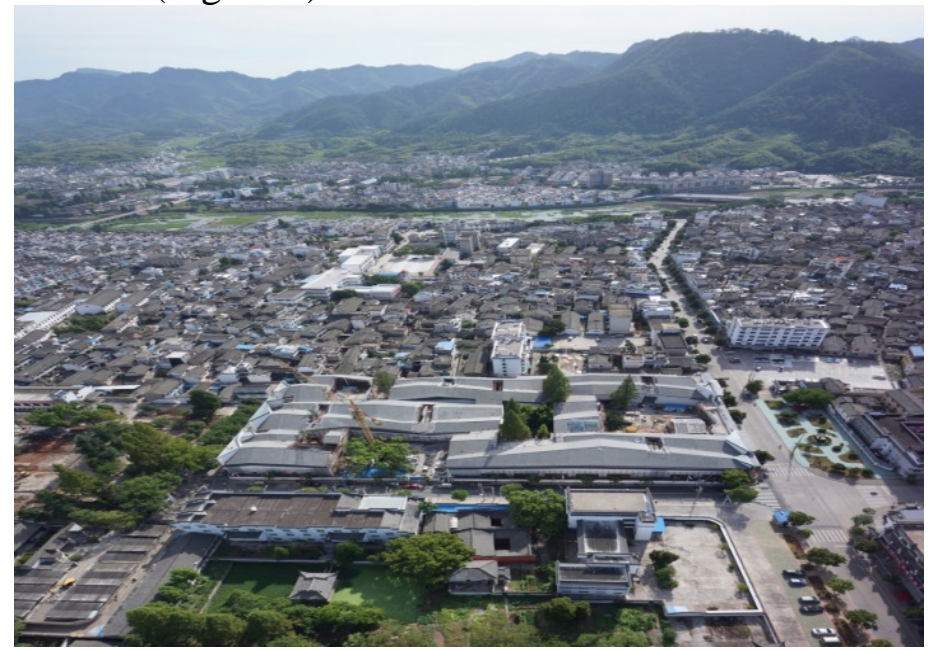

Figure 1 Aerial view of Jixi Museum Picture Source: Internet

Considering that Anhui has a tradition of using natural streams to do things since ancient times, the designers have set up two aqueducts in east and west in the architectural community, along the streets and lanes converging into the water area in the main entrance of the main courtyard, which not only creates the space feeling of Huizhou ancient city, but also conveys the traditional cultural connotation of Huizhou "gathering water is like gathering money". There is an introverted front square on the south side of the building, which accords with the typical layout characteristics of Huizhou folk houses and implies the traditional Chinese idea of "gathering the spirit of geomancy". (Figure 2)

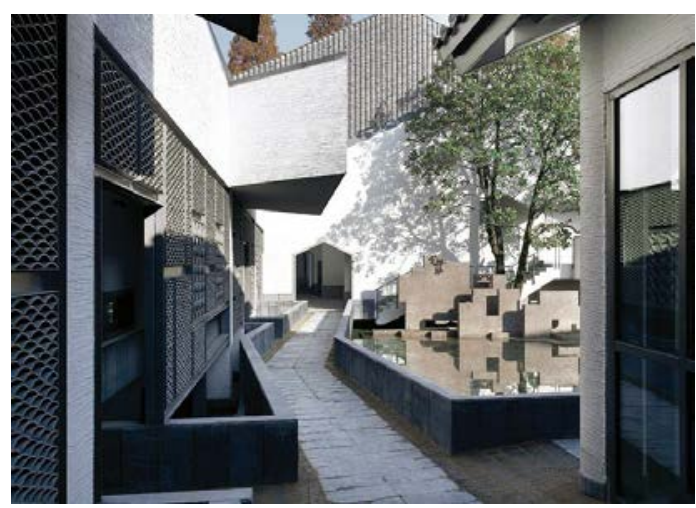

Fig. 2 Courtyard Aqueduct Picture Source: Internet 
The details of the design of Jixi Museum also fully reflect the charm of the Hui style architecture. The triangular roof truss arrangement is full of rhythmical image, and its shape and slope setting both refer to the local buildings. This structure not only adapts perfectly to the zigzag and continuous roof shape, but also integrates into the surrounding ancient city architecture community with its drawn skyline. In terms of the texture of the outer wall of the museum, the designer tries to find a material and color related to the local memory, and forms a special skin texture through modern materials and techniques, which is not just a complete copy of tradition. Instead, through this material, the users can get a sense of historical existence when they see or touch the exterior material, which is the original intention of architectural design.

"White wall and black tiles" has long been a typical feature of Hui style architecture. This kind of white wall is not pure white. As exposed to the sun and rain over the years, the white and gray walls merge with dust, water vapor, etc., which forms a mixture with gray and white with special texture? In the process of creation and construction, designers strive to endow this traditional practice with the expression of contemporary architecture. After trying a variety of materials and practices, the museum's external walls were eventually used light off white elastic textured paint. This waterproof material through a unique manual operation traces, continues the convergence of the artificial and natural attributes of the museum, highlighting the nature of the building as if it grows naturally. The paint uses the texture of water wave and conveys the water-related design concept of Jixi Museum to the visitors.

The museum also uses bricks, tiles and other common local building materials to make them present more modern images. The roof of the Jixi Museum is covered with locally produced tiles from rooftops of various shapes to tortuous ridges. The museum does not completely follow the Huizhou tile roof. While retaining part of the traditional tile practice, it also makes improvement according to the characteristics of modern architectural simplicity. For example, the method of roof ridge does not need the conventional standing tile stacking, but takes the semi-circular imbrex as the roof interlocking tile, highlighting the twists and turns of the roof ridge line simply and clearly. The cornice uses the succinct arc modelling projecting tile of eaves to replace the traditional tiger head dripping tile. The exhibition hall of the museum needs clerestories for daylighting, so there's a fish-scale tile leak under the tile roof, which, on the one hand, extends the roof tile to the facade in a formal way, and on the other hand serves as a shade for the sun-shutter, which can form a more uniform natural lighting effect in the room. In addition, the building also used hollowed-out tile wall as the courtyard wall to distinguish inner and outer space. In addition to using the same fish scale pattern as the tile window, the hollowed-out tile wall is made by inserting part of the rhombus pattern into it to increase the density change of the wall and form a more layered visual effect ${ }^{[5]}$. (Figure 3)

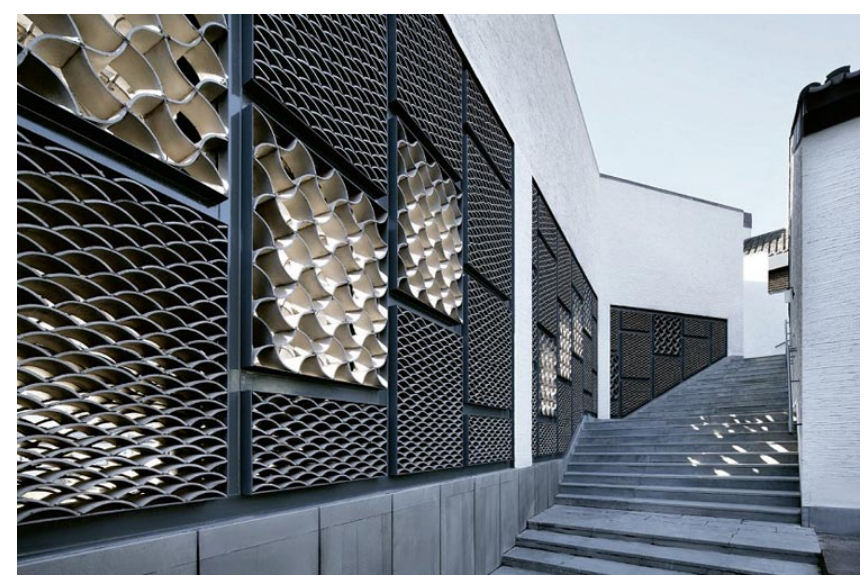

Fig. 3 Hollowed-out Tile Wall Picture Source: Internet 
The Jixi Museum, which emits the temperament of the Jiangnan gardens, combines the local materials with the modern technology and explores the possibility of expressing the traditional intention in the context of contemporary architecture. The creation of the artistic conception of space also makes the museum itself become a huge and intuitive exhibit, making visitors seem to be in the ancient Huizhou ancient town.

\section{The New Suzhou Museum ${ }^{[6]}$ : Expressing Traditional Culture by Architectural Aesthetics Principle and Garden Form}

The New Suzhou Museum is a unique structure. Just like the classical garden city Suzhou, the structure is exquisite, intriguing, and the landscape, flowers and trees and main building with black brick and white wall complement each other. The scope of construction of the new museum lies between the Humble Administrator's Garden ${ }^{[7]}$ and the Prince Zhong Mansion ${ }^{[8]}$, which echoes the former two while maintaining its unique personality and charm. The ancient city of Suzhou is a relatively low housing community as a whole. This natural urban form near the ground reflects the ideology of "harmony of nature and man" in ancient times. As a new building in the old street building group, the new museum has the characteristics of not too high, not too big, or not too outstanding, which integrates perfectly with the surrounding environment. The highest part of the exhibition area in the museum does not exceed two floors, and a portion of the underground space is used as a temporary exhibition hall to meet the needs of the exhibition area.

The overall form and style of the new museum fully reflect the creative techniques of "Chinese style but new style, Suzhou flavor but new materials". "Su" indicates that the architecture combines the traditional features of Suzhou and the humanistic connotation of the city, while "new" is manifested in the selection of novel materials. The architectural material selection is very elegant, replacing the traditional tile with diamond-shaped, black and grayed granite piece, the scrabbling is fixed on the stainless steel plate pitched roof by dry hanging, when wet, it is black, and after drying in the sun, it will turn into dark gray. The roof and wall side decoration of black and gray stone, matched with bright and clean white wall, embodies the traditional black, white and gray colors of the Suzhou style architecture, and adds new connotation to the symbol of the south of the Yangtze River with the white wall and gray tile. (Figure 4)

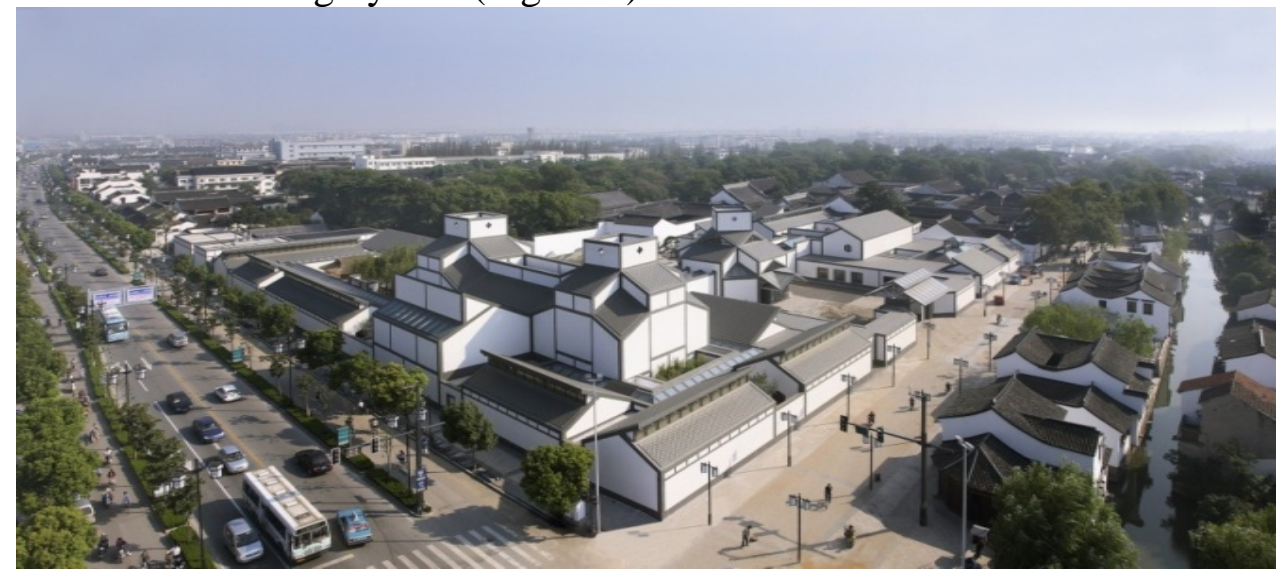

Fig. 4 Aerial View of the New Suzhou Museum Picture Source: Internet

The design of the New Suzhou Museum combines the traditional style of Suzhou architecture and the layout of classical gardens. The architectural layout has a main courtyard and a number of small inner courtyards. The museum is in these courtyards and gardens and is in harmony with the surrounding environment. The main courtyard can be seen as a modern interpretation of the classic Jiangnan garden. The landscape in the courtyard of "using the wall as paper and stone as the 
painting," creates a landscape with great originality and great momentum. The clear outline of the mountain set against the white wall, make the inside garden view echo the Humble Administrator's Garden on the north side, creating an interaction ingeniously ${ }^{[9]}$. In the middle of the different exhibition rooms, a number of small chambers decorated with gardens are separated by walls. Through the orthohexagonal lateral window on the partition wall, we can see the "enframed scenery" and "borrowed scenery" techniques in the traditional garden culture ${ }^{[10]}$, and looking at different angles through the same window also makes people fully appreciate the charm of the classical garden of "scenery changing at every step". There are also many elements in the courtyard that are taken from the traditional gardens and changed, such as rockery, bamboo forest, pavilion, curved bridge, pebbles and so on. The temperament and charm of Chinese traditional culture displayed in the whole architecture come from the traditional Chinese thinking mode of "the unity of nature and man" and "the integration of matter and me", which makes the audience feel the coming natural breath all the time. (Figure 5)

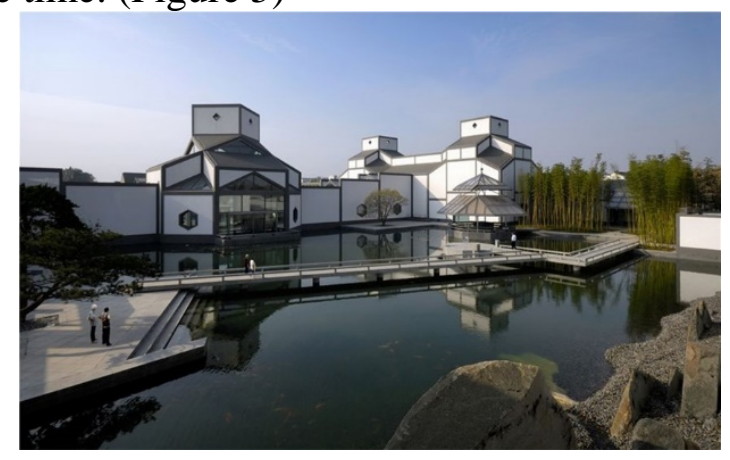

Fig. 5 Main Courtyard Picture Source: Internet

The roof conception of the museum originates from the traditional slope crest landscape in Suzhou. It adopts the modern geometric modeling of sloping roof, which the designer Mr. Ieoh Ming Pei specializes in, and abandons the traditional Suzhou-style beam-frame structure and adopts the modern steel roof structure instead, and the interior of the building is decorated with nostalgic wood structure ceiling. The roof, which combines modern technology and traditional style, not only reflects the sloping roof features of the buildings in the south of the Yangtze River, but also improves the deficiencies of the traditional Chinese "big roof" buildings in natural lighting. The lighting patterns of large exhibition space, independent exhibition area, corridor area and public space have their own characteristics, which not only draw lessons from the Chinese traditional "dormer" techniques, but also have long skylights with metal shading strips, and more creative pyramid glass top window ${ }^{[11]}$. In different indoor spaces, with the movement of the sun, the light passes through the windows of various forms and the shading strips of different patterns, creating ever-changing touching light and shadow. In the natural light, the audience interact with the light and shadow along with the change of time and place, and blend with the natural breath of the building space unconsciously. (Figure 6)

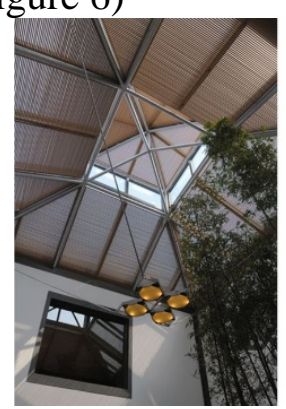

Figure 6 Daylighting Roof

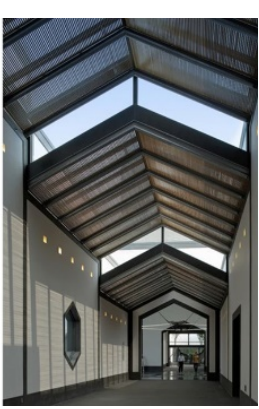

Picture Source: Internet 
The successful design of the New Suzhou Museum proved the possibility of the organic combination of modern and tradition. Through the research and analysis of the new museum architecture, we can feel the wide application of the Chinese traditional culture. The inheritance of history and culture in this building is manifested in the form of gardens, the principles of architectural aesthetics, and the natural spirit of advocating "the unity of nature and man".

\section{Ningbo Museum ${ }^{[12]}$ : "Noe-Nativism" ${ }^{[13]}$ Integrating Local Culture and Cullture}

Ningbo Museum is a comprehensive local museum that shows history, humanities and art. The shape of the architecture if simple but magnificent, and the innovative appearance is rigorous but harmonious. The form of the architecture integrates the local environment, and emphasizes the design language of local flavor and folklore, which presents the design conception of "neo-nativism". The concept of architecture is full of originality. In the process of design and construction, local materials and techniques are used through adjusting measures to local conditions to integrate traditional architectural elements, modern architectural forms and Ningbo regional cultural characteristics to form a strong local atmosphere and local feelings. (Figure 7)

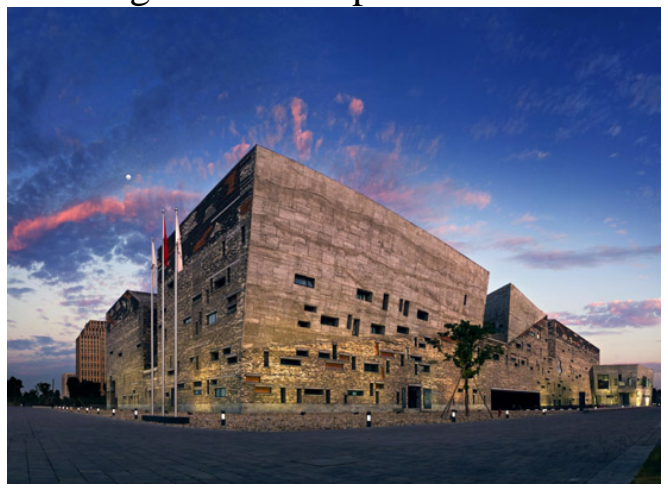

Fig. 7 Ningbo Museum Picture Source: Internet

The design of Ningbo Museum emphasizes the design idea of "mountain", "water" and "ocean". Because of its geographical location, Ningbo is an important port city of "Sea Silk Road". At the same time, the architect also designed the museum as an object with independent life, giving the museum the idea of mountain architecture. The overall form of the architecture is a changing cuboid. From two stories on, the architectural form is split and inclined, and evolves into an abstract "mountain". The five monomers fission from the main body of the building make the public space form a multi-way labyrinth structure and thus create the atmosphere of Ningbo historical and cultural blocks and the garden space experience of one step one scenery. The slightly slanted mountain-like architecture form also creates a tendency to move slightly to the south, and against the water area in the north of the museum, the overall momentum of the building is like a navigating giant ship.

The architectural material selection is the most attractive aspect in Ningbo Museum. Carrying out the designer Wang Shu's natural, economical and environmentally friendly design intention, the "tile chip wall", which accounts for nearly half the area of the museum's exterior facade, is from the previous urban demolition and renovation in Ningbo. Nearly 12000 square meters of walls, using millions of ancient black brick and tiles, is built by hand according to the traditional way as if Ningbo's historical soul has been built into the museum, and the building itself has become part of the historical exhibits of the city (Figure 8). The great significance of "tile chip wall" is that, on the one hand, it embodies the traditional local construction system, which makes the materials and colors of the museum naturally blend into the surrounding environment, while on the other hand, the old bricks and tiles passed down in Ming and Qing dynasties and even in Han and Jin dynasties 
expressed the support of history, witnessed the passing of time in new forms, and fitted the basic idea of "collecting history" of the museum. And "bamboo strip formwork concrete" uses the plant bamboo with Jiangnan charm, innovatively makes the modern hard concrete produce the natural artistic effect. The inclined cast-in-place concrete wall surface uses a special formwork made of bamboo, which presents a natural texture through the texture of bamboo slices after random cracking. The use of "bamboo strip formwork concrete" exterior wall, through bamboo texture, not only softens the rigid and cold feeling that the concrete material usually gives, but also can integrate with "tile chip wall" perfectly ${ }^{[14]}$. (Figure 9)

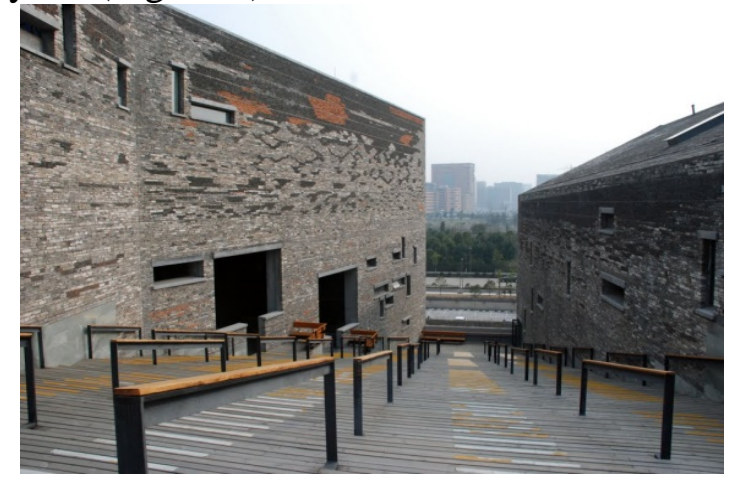

Fig. 8 "Tile Chip Wall" Picture Source: Internet

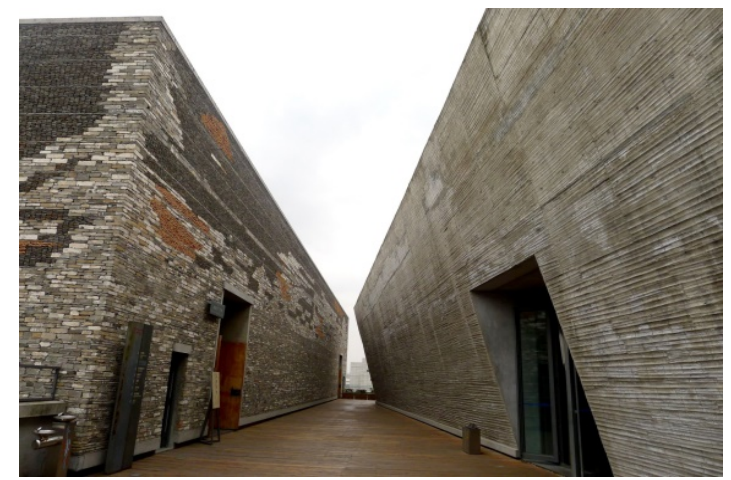

Fig. 9 "Bamboo Strip Formwork Concrete" Picture Source: Internet

Ningbo Museum cleverly makes use of the city's traditional building materials. A large number of recycled materials not only save resources, but also create a strong sense of history and a sense of region through space, material, tone and other details. The innovative and coordinated facade material combination enables the museum to have rich and vivid images and expressions from different distances, reflecting the Chinese traditional virtue of circular construction and presenting the humanistic history and local style of the city at the same time.

Through the analysis of the museums which contain the Chinese traditional culture, we can conclude that the modern architecture can inherit the history humanities mainly through the following design techniques: using the traditional architectural humanistic concept to emphasize the natural view of "the unity of nature and man" and "the integration of matter and me"; using the traditional architectural space design techniques, such as courtyard layout and garden layout, to arouse the reverie and memory of the classical architectural space; using the traditional architectural characteristic and elements to outline people's inner historical and humanistic complex; using traditional building materials to seek recognition and destination of regional spirit and humanistic attributes $^{[15]}$. In the process of actual design creation, we can not only use one of the above design techniques, but also combine several design techniques organically. In short, the birth and development of modern architecture does not mean the disappearance of traditional history 
humanities. The key lies in how to integrate the complex traditional cultural connotation into contemporary architectural art creation with abstract and modern concise design language to create a unique modern architecture with regional, historical, humanistic characteristics.

\section{References}

[1] Liu Yan: Inheritance and Innovation of Modern Architecture Regional Culture, Sichuan Building Materials, 2008, 04, P72-P74

[2] Wang Yuchang. The Role of Museums in Urban Culture, Development, 2010(05), P68-P70

[3] The design was presided over by Li Xinggang, deputy chief architect of the China Institute of Architectural Design and Research.

[4] Hui style architecture is one of the most important schools of Han nationality traditional architecture and an important part of Hui culture. It originated from Dongyang and is a typical representative of Jiangnan architecture.

[5] Li Xinggang, Zhang Yinxuan. Jixi Museum, Jixi, China, World Architecture, 2015(03), P142-P145

[6] Designed by Ieoh Ming Pei, an ethnic Chinese master architect

[7] The Humble Administrator's Garden is the representative work of the Jiangnan classical garden, which is known as China's four most famous gardens together with Beijing the Summer Palace, Emperor Kangxi's Summer Mountain Resort in Chengde, Suzhou Lingering Garden.

[8] Prince Zhong Mansion is the national key cultural relic protection unit, is the largest surviving palace of prince of Taiping Heavenly Kingdom. Li Xiucheng, the Prince Zhong, became the master of the house.

[9] Huang Jianmin, Reverberation and Reproduction-Experiencing the Suzhou Museum Designed by Ieoh Ming Pei and Pei's Architecture Firm, Time Architecture, 2007(03), P64-P75

[10] "Enframed scenery" and "borrowed scenery" are all the architectural art landscape construction methods of Han nationality.

[11] Jin Chengcen, Changing in Control-Design of Roof Lighting (Natural Light) of Suzhou Museum", Packaging World, 2014(05), P89-P91

[12] The designer is the first Pritzker Architecture Award winner in China, and the dean of the College of Architectural Art, China Academy of Fine Arts, Wang Shu

[13] "Neo-nativism" emphasizes the local flavor and nationalization characteristics, and makes use of local materials as far as possible in the design, showing the characteristic of adjusting measures to local conditions.

[14] Yang Xuesong, Li Huiqin. Tradition and Modernity: Feeling the Ningbo Museum. Theoretical Studies on Urban Construction (Electronic Edition), 2013(12)

[15] Wang Yuanlu, Zhang Yajuan. The Application of Chinese Traditional Architectural Elements in Modern Museum Architecture. Shanxi Architecture, 2009(09), P11-P12 